\title{
$\beta$-Decay studies with an implantation technique
}

\author{
J. Büscher ${ }^{\text {a,* }}$, J. Ponsaers ${ }^{\text {a }}$, R. Raabe ${ }^{\text {a }}$, M. Huyse ${ }^{\text {a }}$, P. Van Duppen ${ }^{\text {a }}$, F. Aksouh ${ }^{\text {a,1, }}$, \\ D. Smirnov ${ }^{\text {a }}$, H.O.U. Fynbo ${ }^{\mathrm{b}}$, S. Hyldegaard ${ }^{\mathrm{b}}$, C.Aa. Diget ${ }^{\mathrm{c}}$ \\ ${ }^{a}$ Instituut voor Kem-en Stralingsfysica, K. U. Leuven, Celestijnenlaan 200D, B-3001 Leuven, Belgium \\ ${ }^{\mathrm{b}}$ Institut for Fysik og Astronomi, Århus Universitet, DK-8000 Arhus, Denmark \\ ${ }^{\mathrm{c}}$ Department of Physics, University of York, Heslington, YO10 5DD, UK
}

Available online 6 June 2008

\begin{abstract}
A method is presented for the detection of charged particles emitted in the $\beta$-decay of exotic nuclei. Direct implantation of radioactive nuclei in a very thin double-sided silicon strip detector is now feasible thanks to the advances in the production and post-acceleration of ISOL beams. The high degree of pixelazation, and consequently the small active volume of the detector, and the precise energy of the radioactive ion beam are the key features of the method. Among the advantages of the method are a good precision of the overall normalization and a reduction of the background due to $\beta$-radiation. Several developments are described in the present paper. The detection threshold at low energies is determined using pulsed signals. An alternative energy calibration for the decay events, free from any deadlayer effects can be performed by implanting radioactive ions decaying to known narrow resonances, e.g. ${ }^{20} \mathrm{Na}$. Time and position correlations in a small detection volume can be used to identify possible different decay channels. An overview of precision measurements using this technique is given to prove the strength of the method.
\end{abstract}

(C) 2008 Elsevier B.V. All rights reserved.

PACS: 23.40.-s; 29.40.Wk

Keywords: $\beta$-Decay; Double-sided strip detector; Efficiency; Internal calibration; Correlations

\section{Introduction}

The $\beta$-decay of nuclei close to the drip-lines is in many cases characterised by a large $Q_{\beta}$ value, opening up a variety of decay modes such as $\beta$-delayed particle emission. Some of these decay branches, which often have a small branching ratio $\left(10^{-4}-10^{-6}\right)$, are very sensitive to particular nuclear structure properties. Their study can therefore reveal unique information.

Most measurements so far have employed low energy (typically $60 \mathrm{keV}$ ) beams produced with the isotope separation on-line (ISOL) method, stopped on a thin foil or a tape surrounded by silicon detectors and/or gas counters

\footnotetext{
* Corresponding author.

E-mail address: Jeroen.Buscher@fys.kuleuven.be (J. Büscher).

${ }^{1}$ Present address: CEA/Saclay, Gif-sur-Yvette Cedex, France.
}

$[1,2]$. In these set-ups the $\beta$-delayed ions undergo an energy loss in the implantation foil-, and the dead-layers of the detectors. This can complicate the energy calibration and distort the spectra of the emitted ions, and will affect the minimum threshold energy at which ions can be detected. Furthermore, the low energy part of the $\beta$-delayed particle spectrum can be overwhelmed by the $\beta$-background. Finally, a correct normalization is difficult as the number of implanted atoms and the detection efficiency are difficult to determine with high accuracy.

Implantation of reaction products in double-sided silicon strip detectors (DSSSDs) has been used in a series of experiments dealing with $\alpha$-decay [3], or to investigate properties of nuclei close to the proton drip-line [4]. Specific states of the neutron-deficient nuclei are populated in nuclear reactions. Their characteristic charged particle radioactivity ( $\alpha$-decay, ground-state proton decay and 
$\beta$-delayed proton emission), measured with the DSSSD, is used to tag $\gamma$-ray transitions (recoil decay tagging, RDT). The method was shown to be highly insensitive to $\beta$-particles $[4,5]$.

Some features of the RDT-method can now be used in $\beta$-decay studies when the radioactive beam is directly implanted in the DSSSD. Energetic radioactive ion beams from post-accelerated ISOL facilities or from in-flight facilities have the advantage over conventional ISOL systems in that, using this method, individual decays can be followed.

The general properties of the implantation technique were described in a previous publication [6]; in this paper we report about recent developments made regarding the measurement of very weak $\beta$-delayed charged particle emission channels.

\section{Method}

In order to extract absolute branching ratios, the implantation technique is designed to use the same detector for counting the number of implanted radioactive nuclei and detecting the emitted charged particles. This provides a reliable normalization and a high detection efficiency of the charged particle channels compared to the use of an external source.

Post-accelerated ISOL beams have the advantage to be in many cases pure, intense and well defined in energy. This makes it possible to perform direct measurements of the desired decay channels without any unwanted beam contaminants. For the implantation technique the energy of the beam is tuned in such a way that the ions are stopped around the middle plane of the detector. The beam can be de-focused in order to obtain a rather uniform distribution on the detector surface.

In our experiments we used a DSSSD, manufactured by Micron Semiconductor Ltd, having an active area of $16 \times 16 \mathrm{~mm}^{2}$ and a thickness of $78 \mu \mathrm{m}$. The front face consisted of $48 p+n$ junction strips while the back face had 48 $n+n$ ohmic strips perpendicular to the first ones, defining a total of 2304 pixels. The strip pitch was $335 \mu \mathrm{m}$ while the strip width was $300 \mu \mathrm{m}$. The energy signals and logic signals for each strip were collected using conventional electronics - charge-integrating preamplifiers and shaping amplifiers.

The following beams have already been implanted using this method: ${ }^{6} \mathrm{He}$ and ${ }^{18} \mathrm{Ne}$ at the Cyclotron Research Centre $(\mathrm{CRC})$ in Louvain-la-Neuve, ${ }^{8} \mathrm{Li},{ }^{9} \mathrm{Li}$ and ${ }^{11} \mathrm{Li}$ at TRIUMF in Vancouver, ${ }^{12} \mathrm{~B},{ }^{12} \mathrm{~N}$ and ${ }^{20} \mathrm{Na}$ at the Kernfysisch Versneller Instituut (KVI) in Groningen.

\subsection{Detection efficiency at low energy}

Since the shape of the energy spectrum of the charged particles emitted in the decay contains valuable structure information, it is important to extend the detection to the lowest possible energies. As the radiation is emitted inside the detector's active volume, dead-layers do not play a role and the detection threshold is essentially determined by the electronic noise. The cutoff is not sharp and is determined by the electronic channel; it is thus different for each detector element.

A pulse generator can be employed to measure the detection efficiency at low energy: we sent a known number of signals, at 5 different amplitudes around the limit of detection, to the preamplifiers. The signals generating a trigger were recorded, producing peaks in the energy spectra corresponding to the pulser amplitudes, see Fig. 1(left) for an example. For each strip the detection efficiency was calculated by fitting the peak areas as a function of energy using a Fermi function with 2 parameters (Fig. 1(right)): the slope of the function and the energy at 50\% efficiency. The total efficiency curve of the detector was constructed as a weighted mean of all the fits on the strips, where the weight was given by the number of ions implanted in the strip during an actual measurement. We applied this procedure during our experiments, obtaining thresholds ranging from $200 \mathrm{keV}$ to $400 \mathrm{keV}$, mainly due to the different amplification settings used in each case.

\section{2. $\beta$-Suppression}

At low energies, apart from any electronic noise considerations, one has to deal with the huge background arising
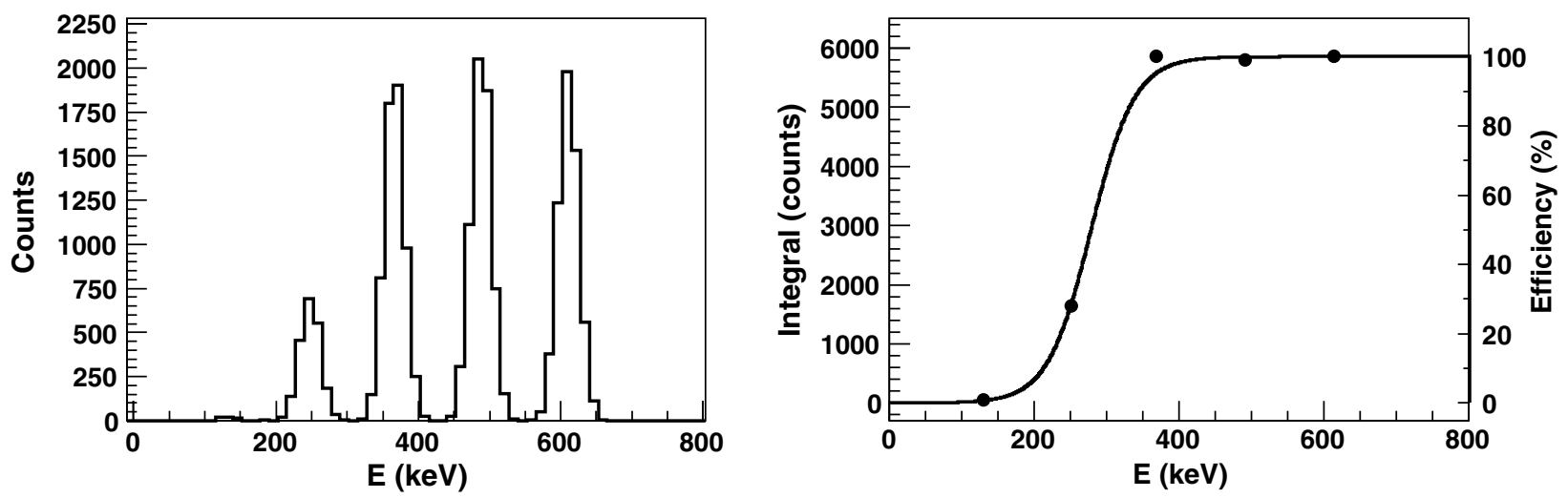

Fig. 1. Left: pulsed signal counts in one strip as a function of energy. Right: integrated peak areas as a function of energy fit by a Fermi function, defining the detection efficiency at low energies. 
from $\beta$-particles. By segmenting the detector into pixels (requiring only one signal in a strip on each face) the influence of the $\beta$-particles can be strongly reduced, since the maximum energy deposited in a pixel can be very small. Further reduction of the $\beta$-background can be achieved by setting a maximum limit for the energy difference between the strip signals from the two sides of the detector [6]. This way it is possible to eliminate those $\beta$-particles, which diffuse mainly along one strip on one side depositing a large fraction of their energy, at the same time generating only a small signal above the threshold in one of the strips on the other side.

The $\beta$-suppression factor $\mathscr{F}(E)$ is defined as the ratio between the total emitted $\beta$-particles and those detected above the energy $E$. We measured $\mathscr{F}(E)$ in a series of cases, presented in Fig. 2. The ${ }^{18} \mathrm{Ne}$ nucleus is a pure $\beta^{+}$emitter, with particles up to $Q=3.4 \mathrm{MeV}$; we implanted it in our DSSSD with a short (1.5-1.5 s) on/off beam modulation, in order to obtain a pure decay spectrum during the beam-off periods. The spectrum is presented in Fig. 3, and contains signals originating from the decays of ${ }^{18} \mathrm{Ne}$ and its daughter ${ }^{18} \mathrm{~F}\left(Q_{\beta^{+}}=0.6 \mathrm{MeV}\right)$. The factor $\mathscr{F}(E)$ was calculated from the number of detected events, knowing the total number of implanted ions and the fraction of expected decays due to the observation window. The calculation was also performed for the full statistics, which served to check the contribution of ${ }^{18} \mathrm{~F}$ (different in the two cases because of its half-life, much longer than that of ${ }^{18} \mathrm{Ne}$ ); in this case, the spectrum of the $\beta$-particles was fitted with an exponential decay in order to subtract the contribution due to low energy events from scattered beam particles. The same procedure was adopted with the data collected from the decay of the ${ }^{6} \mathrm{He}$ nuclei $\left(Q_{\beta^{-}}=\right.$ $3.5 \mathrm{MeV})$, and those from the decay of ${ }^{11} \mathrm{Be}\left(Q_{\beta^{-}}=\right.$ 11.5 MeV); the latter were obtained by implanting ${ }^{11} \mathrm{Li}$ nuclei with a long beam on/off modulation (20-20 s), which

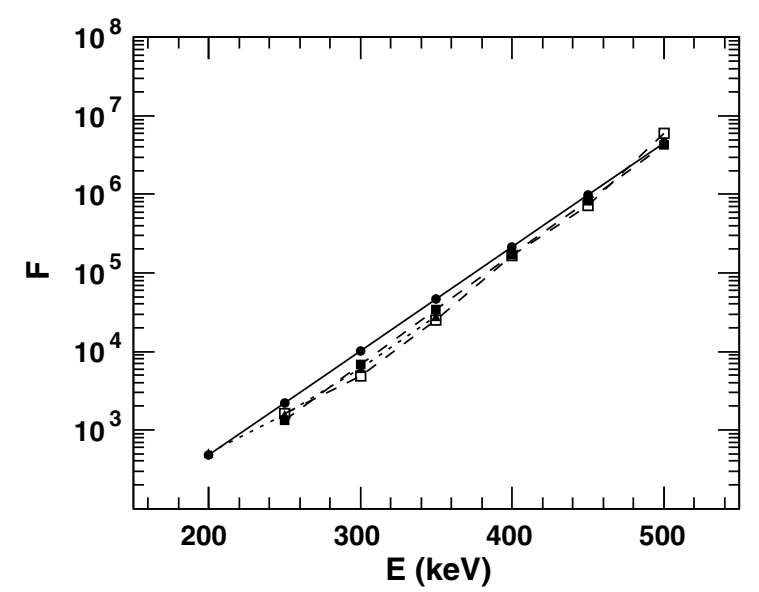

Fig. 2. $\beta$-suppression factor $\mathscr{F}$ as a function of energy. Dots: ${ }^{6} \mathrm{He}-$ experiment; squares: ${ }^{18} \mathrm{Ne}$-experiment, filled squares corresponding to the full statistics and empty squares to beam-off events; triangles: ${ }^{11} \mathrm{Li}-$ experiment, $\beta$-particles emitted in the decay of ${ }^{11} \mathrm{Be}$.

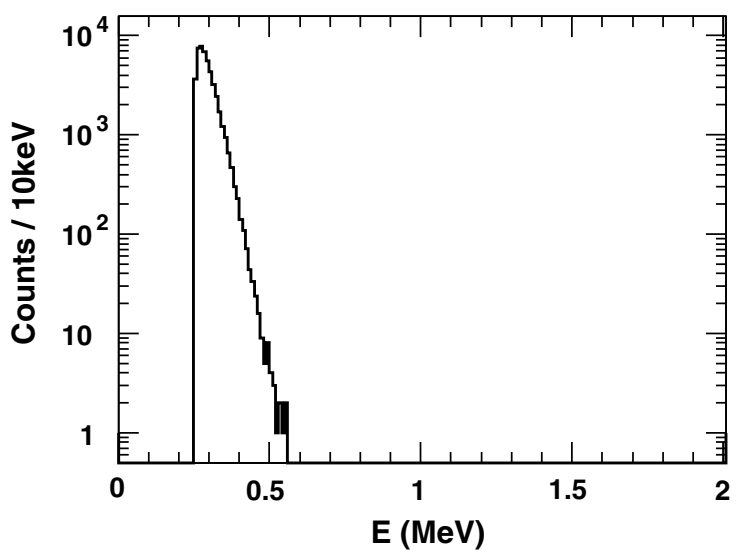

Fig. 3. Spectrum of detected events following the implantation of ${ }^{18} \mathrm{Ne}$, and during the $1.5 \mathrm{~s}$ beam-off period when only $\beta^{+}$-particles are expected.

produced, after a few seconds in the beam-off periods, a pure ${ }^{11}$ Be-decay spectrum.

As shown in Fig. 2, the measurements gave similar results. The data were collected from very different amounts of the total activity (from $4.61 \times 10^{8}$ implanted ions for ${ }^{6} \mathrm{He}$ to $1.16 \times 10^{7}$ for ${ }^{11} \mathrm{Be}$ ) and electronic amplifications, which induced different detection thresholds. Since no corrective factors were introduced, we conclude that the $\beta$-suppression factor in our DSSSD is substantially independent from the end-point energy of the decay, reaching about $10^{7}$ for signals recorded beyond $500 \mathrm{keV}$.

\subsection{Reconstruction of ion-emission events}

By considering only single-pixel events and a limited strip energy difference, $\beta$-particles events are strongly suppressed. For the emitted ions, the generation of a signal in one single-pixel depends on the energy and type of the ions and the dimensions of the DSSSD. The properties of the DSSSD should be chosen in such a way that the ranges of emitted ions are substantially smaller than the dimensions of one pixel. Simulations can be performed to evaluate the actual response of the detector. In our case, the simulation of $\alpha+\mathrm{d}$ events emitted in the $\beta$-decay of ${ }^{6} \mathrm{He}$ [6] showed that virtually all $(99.94 \%)$ events above the detection threshold are confined within one pixel, assuming a homogeneous implantation distribution. The efficiency for the detection of the decay events was therefore only limited by the possible dead-time of the acquisition system and could be determined accurately.

Another effect influences the identification of events in the detector. When the implantation takes place in the inter-strip area, its large energy deposition may generate signals in two neighboring strips on the same side, both above the detection threshold. The weaker signal from the decay would be split in the same way, but it may happen that only one part is above the threshold. In these cases, a reconstruction of the implantation event was made by adding the two energy signals and assigning the summed 
energy to one strip (the one with the higher signal) to recover the correct normalization. This method becomes important when one makes use of the correlation between the implantation and subsequent decay events in the same pixel (see below Section 2.5).

\subsection{Energy calibration}

Usually the energy calibration of silicon detectors is performed using an external source emitting $\alpha$-particles of known energies. Sources are available commercially in the energy range between $3 \mathrm{MeV}$ and $6 \mathrm{MeV}$; in this region the calibration will have the best precision, usually better than $0.1 \%$ for a detector with a resolution around $30 \mathrm{keV}$. However, if an accurate measurement is needed, a correction for the dead-layer present on the surface of silicon detectors has to be added: for example, in a typical aluminium layer of $0.5 \mu \mathrm{m}, \alpha$-particles of $5 \mathrm{MeV}$ energy lose about $80 \mathrm{keV}$ [7]. The energy loss depends on the type and energy of particles, and becomes especially important at low energies.

The energy of particles before they penetrate into the detector has to be calculated using energy loss tables, once the incident angle of the particle and the thickness of the dead-layer are known. The latter can be determined, by placing a source at different angles relative to the detector plane and measuring the shift in the position of the energy peaks. We adopted such a procedure, recording the spectra produced by an $\alpha$-source placed at $90^{\circ}$ and $22^{\circ}$ with respect to the plane of our DSSSD. From the difference in the position of the energy peaks, an energy loss of 45(1) keV was found, corresponding to a thickness of $0.340(3) \mu \mathrm{m}$ of silicon equivalent.

Such a method suffers from the uncertainties propagating from the two calibration measurements, and of course relies on the accuracy of energy loss tables. Another possibility is present, offered by the availability of radioactive ion beams, if the aim of an experiment is to measure the

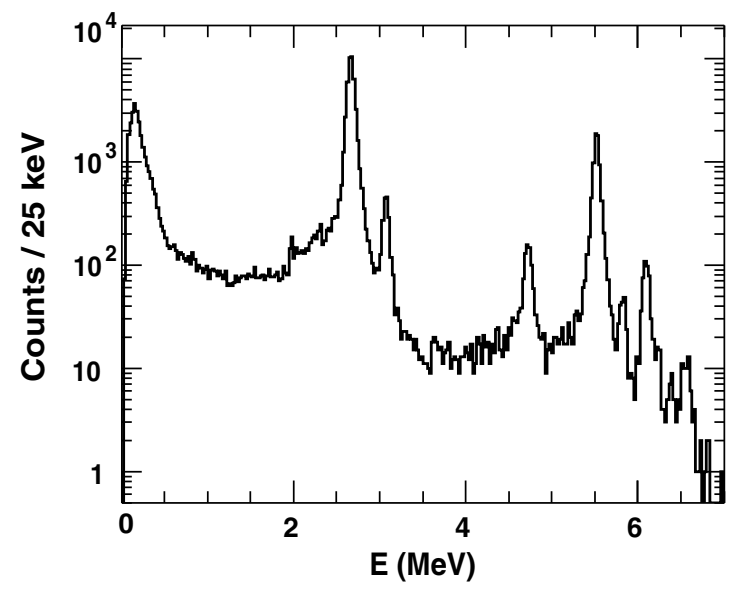

Fig. 4. Spectrum of the charged particles emitted in the $\beta$-decay of ${ }^{20} \mathrm{Na}$ nuclei implanted in the DSSSD. The peaks correspond to unbound states in ${ }^{20} \mathrm{Ne}$ above the $\alpha$-emission threshold. energy of events originating in the detector itself. An internal calibration can be performed by directly implanting unstable ions, which decay emitting charged particles at sharp energies. The ${ }^{20} \mathrm{Na}$ nucleus (with a half-life of $448(2) \mathrm{ms})$ undergoes $\beta^{+}$-decay towards ${ }^{20} \mathrm{Ne}$, with about a $20 \%$ branching ratio towards narrow unbound states, that in turn decay emitting an $\alpha$-particle. The method has been used in [8], and we adopted it in the occasion of the measurements at the KVI facility in Groningen, obtaining the spectrum shown in Fig. 4. The peaks range between $2.5 \mathrm{MeV}$ and $6.5 \mathrm{MeV}$ and have widths essentially determined by the detector's intrinsic resolution. This method relies on a single calibration and avoids the correction for the dead-layer; however, the effect of $\beta$-summing has to be taken into account, since the total energy deposited in a pixel is measured. This information is provided by simulations of the energy loss of $\beta$-particles in the DSSSD.

\subsection{Time and position correlation}

If the implanted ion has only one decay channel, the beam is tuned in on- and off-periods to obtain a clean energy spectrum. Fig. 3 shows the pure $\beta$-decay spectrum of ${ }^{18} \mathrm{Ne}$, recorded during the beam-off periods: this demonstrates that no other events are recorded above the $\beta$-particle signals, making it possible to measure ion-emission spectra from very weak decay channels as in the case of ${ }^{6} \mathrm{He}$. When more decay channels are open, the identification is based on the spectrum of the emitted particles, on the timing behavior of the radiation, and on correlations between the implantation signal, the decay signature, and possible further signals from daughter-decay. Both time and position correlations can be applied: the latter is based on the segmentation of the detector, as the daughter nuclei remain well-localized in the detection element where the mother nucleus was implanted. Time correlations are made acquiring a time stamp for each event. In this case continuous beam implantation can be used.

If the beam rate is high, a second ion can be implanted after a first implantation before all decays take place. To avoid this, a first rule of thumb for the choice of the beam rate is having the beam rate per pixel lower than the decay rate of the longest living daughter nucleus.

Specific precise corrections can be calculated if the implantation rate in one single-pixel, $\lambda_{\mathrm{I}}$, and the decay rates are known. When an ion is implanted at time $t_{0}=0 \mathrm{~s}$ :

(i) The probability density function, $p_{\mathrm{M}}\left(t_{1}\right)$, to observe the mother decay at time $t_{1}$ before a second implantation occurs, can be written in the following form:

$p_{\mathrm{M}}\left(t_{1}\right)=\lambda_{\mathrm{M}} \mathrm{e}^{-\lambda_{\mathrm{M}} t_{1}} \times \mathrm{e}^{-\lambda_{1} t_{1}}$,

where $\lambda_{\mathrm{M}}$ is the decay rate of the mother nuclei. The corresponding total probability is found integrating over $t_{1}$ from 0 to $+\infty$ :

$P_{\mathrm{M}}=\frac{\lambda_{\mathrm{M}}}{\lambda_{\mathrm{M}}+\lambda_{\mathrm{I}}}$. 
(ii) If also the daughter nucleus is unstable, the probability density function, $p_{\mathrm{D}}\left(t_{1}, t_{2}\right)$, to have a daughterdecay at time $t_{2}$ after the mother decay at time $t_{1}$ without a second implantation event is

$p_{\mathrm{D}}\left(t_{1}, t_{2}\right)=\lambda_{\mathrm{M}} \lambda_{\mathrm{D}} e^{-\left(\lambda_{\mathrm{M}}-\lambda_{\mathrm{D}}\right) t_{1}} \mathrm{e}^{-\left(\lambda_{\mathrm{D}}+\lambda_{\mathrm{I}}\right) t_{2}}$,

with $\lambda_{\mathrm{D}}$ the decay rate of the daughter nuclei. In this case the probability, $P_{\mathrm{D}}$, is found after integration over $t_{2}$ from $t_{1}$ to $+\infty$, followed by the integration over $t_{1}$ from 0 to $+\infty$ :

$P_{\mathrm{D}}=\frac{\lambda_{\mathrm{D}}}{\lambda_{\mathrm{D}}+\lambda_{\mathrm{I}}} \times \frac{\lambda_{\mathrm{M}}}{\lambda_{\mathrm{M}}+\lambda_{\mathrm{I}}}$.

The correlation technique has been used to identify the ${ }^{9} \mathrm{Li}+\mathrm{d}$ and ${ }^{8} \mathrm{Li}+\mathrm{t}$ channels in the $\beta$-decay of ${ }^{11} \mathrm{Li}\left(T_{1 / 2}=\right.$ $8.59 \mathrm{~ms}$ ), which was done for the first time during a measurement at TRIUMF. A correction for the branching ratio could be estimated using Eq. (4).

\section{Results}

Using the implantation technique we have performed the measurement of the $\beta$-decay of several unstable nuclei, among which the halo-nuclei ${ }^{6} \mathrm{He}$ and ${ }^{11} \mathrm{Li}$. Future plans include the measurement of the decay of ${ }^{8} \mathrm{~B}$ to obtain information about its neutrino spectrum.

The branching ratio (BR) of the $\beta$-delayed $\alpha+\mathrm{d}$ emission in the ${ }^{6} \mathrm{He}$ decay was measured at the CRC in Louvain-la-Neuve, Belgium [6] with high precision; BR = $(1.65 \pm 0.1) \times 10^{-6}$ above the energy threshold $E_{\text {c.m. }}=$ $525 \mathrm{keV}$. The summed energy spectrum of the $\alpha+\mathrm{d}$ particles has also been obtained [9].

The identification of the ${ }^{9} \mathrm{Li}+\mathrm{d}$ and ${ }^{8} \mathrm{Li}+\mathrm{t}$ channels in the ${ }^{11} \mathrm{Li}$ decay was achieved and the branching ratio and energy spectrum of both channels were measured. We also obtained the energy spectra for both ${ }^{8} \mathrm{Li}$ and ${ }^{9} \mathrm{Li}$ nuclei.

Excited states in the ${ }^{12} \mathrm{C}$ nucleus above the triple $\alpha$-threshold were investigated by implanting both ${ }^{12} \mathrm{~B}$ and ${ }^{12} \mathrm{~N}$ beams at the KVI facility in Groningen, The Netherlands. The $0^{+}$Hoyle state at $7.65 \mathrm{MeV}$ in ${ }^{12} \mathrm{C}$, just $0.3 \mathrm{MeV}$ above the break-up threshold, has been observed and the branching ratios for $\beta^{+}$- and $\beta^{-}$-decay to the states in the $3 \alpha$-continuum were revised, with a precision improved by a factor 10 [10].

\section{Conclusions and outlook}

The implantation technique using a segmented silicon detector is discussed with respect to its use in measurements of $\beta$-delayed charged particle emission.

Due to the $\beta$-suppression and the high detection efficiency for ions, precise branching ratios and energy spectra down to low energies can be obtained. The possibility of an internal energy calibration, and the use of time and position correlation to identify different decay channels are described.

Further aspects can be considered for the improvement of the method. Auxiliary $\beta$ - and $\gamma$-detectors as well as $\Delta E$-detectors can be added to the set-up to further distinguish between different signals. The possibility of cooling the DSSSD can result in a better resolution and reduction of the leakage current together with more stable conditions.

\section{References}

[1] K. Riisager, M.J.G. Borge, H. Gabelmann, P.G. Hansen, L. Johannsen, B. Jonson, W. Kurcewicz, G. Nyman, A. Richter, O. Tengblad, et al., Phys. Lett. B 235 (1990) 30.

[2] M.J.G. Borge, L. Johannsen, B. Jonson, T. Nilsson, G. Nyman, K. Riisager, O. Tengblad, K. Wilhelmsen Rolander, and the ISOLDE Collaboration, Nucl. Phys. A 560 (1993) 664.

[3] R.D. Page, P.J. Woods, R.A. Cunningham, T. Davinson, N.J. Davis, A.N. James, K. Livingston, P.J. Sellin, A.C. Shotter, Phys. Rev. C 49 (6) (1994) 3312.

[4] E.S. Paul et al., Phys. Rev. C 51 (1) (1995) 78.

[5] P.J. Sellin et al., Nucl. Instr. and Meth. A 311 (1992) 217.

[6] D. Smirnov, F. Aksouh, S. Dean, H. De Witte, M. Huyse, O. Ivanov, P. Mayet, I. Muhka, R. Raabe, J.-C. Thomas, et al., Nucl. Instr. and Meth. A 547 (2005) 480.

[7] J.F. Ziegler, J.P. Biersack, U. Littmark, The Stopping and Range of Ions in Solids, Pergamon Press, New York, 1985.

[8] W.T. Winter, S.J. Freedman, K.E. Rehm, J.P. Schiffer, Phys. Rev. C 73 (2006) 025503.

[9] R. Raabe, J. Büscher, J. Ponsaers, et al., Phys. Rev. C, submitted for publication.

[10] S. Gadegaard Pedersen et al., Proceedings of Science, NIC-IX, 2006, p. 244. 